\title{
Microbial dormancy and boom-and-bust population dynamics under starvation stress
}

\author{
A. C. Fowler ${ }^{1,2}$ and H. F. Winstanley ${ }^{1}$ \\ ${ }^{1}$ MACSI, University of Limerick, Limerick, Ireland \\ ${ }^{2}$ OCIAM, University of Oxford, Oxford, UK
}

January 20, 2018

\begin{abstract}
We propose a model for the growth of microbial populations in the presence of a rate-limiting nutrient which accounts for the switching of cells to a dormant phase at low densities in response to decreasing concentration of a putative biochemical signal. We then show that in conditions of nutrient starvation, selfsustained oscillations can occur, thus providing a natural explanation for such phenomena as plankton blooms. However, unlike results of previous studies, the microbial population minima do not become unrealistically small, being buffered during minima by an increased dormant phase population. We also show that this allows microbes to survive in extreme environments for very long periods, consistent with observation. The mechanism provides a natural vehicle for other such sporadic outbreaks, such as viral epidemics.
\end{abstract}

Keywords: dormancy, survival, bloom, oscillations, mathematical model.

\section{Introduction}

In this paper we address a question which sometimes arises in population biology, particularly when populations oscillate. Such situations are commonly modelled by a set of differential equations, and it is not unusual for the resulting calculated populations to reach very low levels; so low, in fact, that in reality the population would go extinct (Campillo and Lobry 2012). Examples of this are the study by Murray et al. (1986) on recurrent fox rabies outbreaks, which was criticised by Mollison (1991) for precisely this reason (the 'atto-fox' effect, cf. Cruickshank et al. (1999) and Renshaw (2005)). Models of the immune response commonly exhibit such features (Dibrov et al. 1977a,b, Fowler 1981) (perhaps because extinction of antigens is desirable), as do population models with delay (Fowler 1982, Fowler and Mackey 2002), and more generally, those exhibiting 'boom-and-bust' dynamics, including plankton blooms (Huppert et al. 2005, Mahadevan et al. 2012), glycolitic oscillations (Goldbeter 1996) and oceanic calcifiers (Omta et al. 2013). 


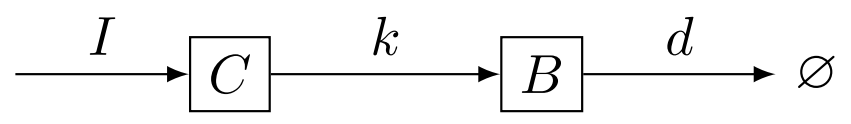

Figure 1: Schematic of simple biomass/nutrient dynamics. $B$ represents (microbial) biomass, while $C$ represents nutrient, for example organic carbon $\mathrm{CH}_{2} \mathrm{O}$. $I$ is the prescribed input rate of nutrient, $k$ the rate constant in converting nutrient to biomass, and $d$ is the microbial death rate.

A number of the above studies focus on models which represent the simple interaction between a population and its rate-limiting nutrient. These include the models of Omta et al. (2013), Huppert et al. (2005), and Goldbeter (1996), and the same basic model is used in a microbial context by Sanchez-Vila et al. (2013). It is in this latter context that our interest lies. To be specific, the model structure of interest is that shown in figure 1. It represents an input of nutrient $C$ (for example, in a chemostat) which feeds a microbial population $B$, which undergoes growth as well as death. The resulting mathematical model takes the form

$$
\begin{aligned}
\dot{B} & =k Y C B-d B \\
\dot{C} & =I-k C B
\end{aligned}
$$

where for simplicity we ignore Monod kinetics (which in any case has a cosmetic effect, which we discuss in further detail later). In these equations, $k$ is a rate constant, $Y$ is a yield coefficient, $d$ is death rate, and $I$ represents the nutrient input rate.

Some comments on the choice of a constant nutrient supply $I$ should be made. In a bacterial growth experiment in a petri dish, there is no supply, but an initial rich nutrient concentration is given. A more environmentally realistic experiment is the chemostat reactor, in which nutrient is supplied in the input liquid feed, as in (1.1), but is limited by the drainage from the reactor, even in the absence of bacterial consumption. The equivalent of a chemostat in a soil system is, for example, supply of oxygenated rainwater or other nutrient supply, e.g., wastewater, and its removal to an underlying saturated aquifer. Particularly in wastewater systems, it is typically the case that microbial consumption removes all the nutrient, and this underpins the neglect of a sink term of the form $-C / \tau$ in (1.1), where $\tau$ would be a residence time for the system. Our neglect of such a term, if relevant, is equivalent to an assumption that the residence time is much longer than the dynamical time scales of interest.

The dynamics of the model (1.1) are simply studied, see for example Omta et al. (2013). There is a steady state which is stable but excitable, particularly when the supply of nutrient is small. It is in this context that transient oscillations occur, in which temporal spikes of biomass growth occur at regular intervals, separated by periods where the biomass becomes extremely small. The reason for this striking behaviour can be found by an asymptotic analysis of the model, as was demonstrated by Fowler (2013). In further work, Fowler et al. (2014) extended the model to a system of competing microbial populations, and showed that self-sustained oscillations occur in conditions of starvation, and these solutions were analysed by Fowler (2014). 
Again, the minima in the microbial populations are so low that in practice, actual extinction would occur. The use of the word 'starvation' in the present context does not necessarily connote a complete absence of nutrient, but refers to conditions in which a suitable comparative measure of the input is small. For (1.1), this takes the form

$$
I \ll d / k Y
$$

in the present context, 'starvation' is further described below.

And yet, as we know, bacteria can be found in the most inhospitable environments. According to Kirchman $\left(2012\right.$, p. 9), while normal soils may contain $10^{9}$ cells $\mathrm{g}^{-1}$, even in deep environments kilometres below the Earth's surface, there may be of the order of $10^{3}$ cells $\mathrm{g}^{-1}$ which are viable. This is in contrast to the implication of the simple model (1.1) and its more sophisticated variants, which seem to imply that in starvation conditions, microbial extinction would occur in practice. Simply, if $C$ is too low, (1.1) implies that $B \rightarrow 0$. And yet, it is suggested that the 'normal' states for many environmental bacteria are similar to starvation conditions (Roszak and Colwell 1987).

It is this conundrum that we wish to address, in the context of (1.1). In one sense, the answer is obvious. If bacteria remain viable in starvation conditions, then they must be in a dormant state (Stevenson 1978, Kaprelyants et al. 1993), where the normal processes of respiration and growth (and death) are stalled. Dormancy is widely seen in the bacterial world (Lennon and Jones 2011) in a variety of forms which allow populations to persist through environmental challenges, even over geological timescales (Hoehler and Jørgensen 2013). Dormancy is increasingly viewed as an important evolutionary hedging strategy for survival (Shoemaker and Lennon 2017, Locey et al. 2017), with recognised importance in medicine (Rittershaus et al. 2013, Harms et al. 2016, Lipworth et al. 2016), and soil systems and ecosystem modelling (Wang et al. 2014, He et al. 2015, Joergensen and Wichern 2018). Various terms describe the different manifestations and types of dormancy in different fields: sporulation, latency, quiescence, persisters. The common trait is the ability to switch between an active form and a hardy, relatively inactive form which can survive and subsequently reactivate or reseed an active population. This necessitates the introduction of a second microbial population $A$, in which respiratory and growth processes are absent. Our basic hypothesis is that at low population levels, a switch occurs, whereby active microbes switch from their normal birth/death cycle to a state of hibernation, from which they can be aroused in the presence of excess nutrient. This description of the switching process is very similar to the description of the blood cell growth model pioneered by Mackey and co-workers (Mackey 1978, Haurie et al. 1998).

There have been a number of other mathematical models which have been proposed to study the process of dormancy. Bär et al. (2002) modelled the response to intermittent water stress in drylands, comparing bacterial populations without a dormant state to those with the ability to enter and recover from a dormant state based on water availability alone. Malik and Smith (2006) modelled distinct active and dormant populations in a continuous flow planktonic culture, with nutrient-dependent 


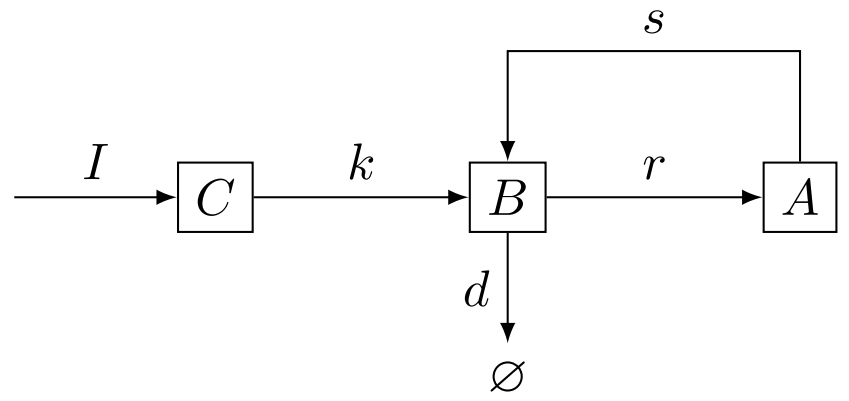

Figure 2: Schematic of the dormancy model. $B, C, I, k, d$ have the same meaqnings as in figure $1, A$ denotes microbes in the dormant state, and $r$ and $s$ denote statedependent switching rates between active $(B)$ and dormant $(A)$ states.

dormancy entry and exit rate functions. They noted oscillatory transients in the approach to the survival steady state, but the model did not show sustained oscillatory solutions. Malik and Smith (2008) looked at the response of dormancy-capable populations to periodic and random switching between favourable and adverse conditions. Hadeler (2013) aimed to place a simple model of dormancy in a broader context of ecological models, analysing the effect of dormancy on periodic orbits in excitable systems. Ayati (2012) modelled bacterial dormancy strategies in batch culture with periodic partial reculturing into fresh media. He proposed a maturation model of dormancy under nutrient stress, in which reactivation of dormant cells occurs only when they have consumed a fixed amount of nutrient, which they are only able to do when nutrient concentration is above a threshold level. Ayati and Klapper (2012) applied a similar model to compare dormancy in chemostat suspended cultures with spatially-structured biofilm comprising active, dormant, and dead cells with nutrient supplied by diffusion from the free biofilm surface.

Mathematical modelling of dormancy is well-established (e.g., Wang and Levin 2009, Stolpovsky et al. 2011, 2015). One possibility (Jones and Lennon 2010) is to associate a switch from active to dormant states with the level of available nutrient. In our study, we will examine several possible forms for the functions governing awakening and hibernation, with a view to finding one which allows boom and bust dynamics, without exposing the population to extinction. The mathematical model which represents these ideas is presented in the following section.

\section{Mathematical model}

The modification of the schematic in figure 1 which describes our hypothesised microbial switching is shown in figure 2. The additional processes are a transformation (hibernation) of $B$ to $A$ by a cell-specific rate $r$, and the re-activation (awakening) of $A$ at a cell-specific rate $s$. The switching between active and dormant states is described by modulating the rates $s$ and $r$ by awakening and hibernation functions $p$ and $q$, respectively, each of them $\in(0,1)$, and which can be thought of as cell-specific 
probabilities of switching mode. We will examine various different combinations of functional dependence. For example, the assumption of a nutrient-dependent switching (Jones and Lennon 2010) would suggest $p$ and $q$ are respectively increasing and decreasing functions of $C$; if paracrine signalling enabled the switches, then we would take $p$ and $q$ as functions of $B$. To indicate the fact that $p$ and $q$ are not constant, we write them as $p(U)$ and $q(V)$, where $U$ and $V$ denote the switching variables, which in our models are assumed to be either $B$ or $C$.

The model describing the dynamics is thus given by

$$
\begin{aligned}
\dot{B} & =k Y C B-d B-r q(V) B+s p(U) A, \\
\dot{C} & =I-k C B \\
\dot{A} & =r q(V) B-s p(U) A,
\end{aligned}
$$

and we suppose that the switching functions define a level of cell concentration $K$ at which the switch takes place. This is elaborated below, as its value will depend on the choices of the functions $p$ and $q$.

We non-dimensionalise the model in a way motivated by our previous work (Fowler 2013), by scaling the variables as

$$
A \sim B \sim K, \quad C \sim \frac{d}{k Y}, \quad t \sim \frac{1}{\sqrt{d k K}}
$$

and the scaled equations take the form

$$
\begin{aligned}
\varepsilon \dot{b} & =(c-1) b-\mu q b+\lambda p a, \\
\dot{c} & =\varepsilon(F-c b), \\
\varepsilon \dot{a} & =\mu q b-\lambda p a,
\end{aligned}
$$

where the lower case signifies the scaled variables, and the parameters are defined by

$$
\varepsilon=\sqrt{\frac{k K}{d}}, \quad F=\frac{I Y}{d K}, \quad \mu=\frac{r}{d}, \quad \lambda=\frac{s}{d} .
$$

The physical interpretations of these dimensionless parameters is as follows: $\mu$ and $\lambda$ are measures of the hibernation and reactivation rates relative to the microbial death rate, $F$ measures the size of the nutrient supply, and the important parameter $\varepsilon$ represents the rate of microbial growth and death rates relative to the rate of nutrient consumption. As mentioned, the choice of scale $K$ for $B$ and $A$ depends on the choice of the switch functions, and is discussed further below. We will suppose that the time scales associated with cellular processes are comparable, suggesting $\mu, \lambda \sim O(1)$; the basic assumption that the switching process is activated at low population levels implies $K$ is 'small', which we interpret by assuming that $\varepsilon \ll 1$; the value of $F$ is associated with the richness of the nutrient supply. We will use these indicators to guide our choice of parameters below. 


\subsection{Choice of switch functions}

We define a Hill function for arousal

$$
R(x)=\frac{x^{m}}{1+x^{m}}
$$

a complementary function for hibernation

$$
H(x)=\frac{1}{1+x^{n}}
$$

and a modified arousal function

$$
R^{*}(x)=\frac{\delta+x^{m}}{1+x^{m}}
$$

and we have used combinations of these to study the dynamics of the model. These are detailed in the following section. Generally, we take $p=R(x)$ or $R^{*}(x)$ and $q=H(y)$, with $x$ and $y$ independently being either $c$ or $b$. When a dependence on $B$ is assumed, the dimensional form of the Hill function is

$$
R_{B}(B)=\frac{B^{m}}{K_{B}^{m}+B^{m}},
$$

and then our choice of the scale $K$ in $(2.2)$ is $K=K_{B}$. On the other hand, if the Hill function is dependent on $C$, i. e.,

$$
R_{C}(C)=\frac{C^{m}}{K_{C}^{m}+C^{m}}
$$

then the corresponding dimensionless form is

$$
\frac{c^{m}}{\kappa^{m}+c^{m}}=R\left(\frac{c}{\kappa}\right)
$$

where

$$
\kappa=\frac{k Y K_{C}}{d}
$$

and we have the freedom to choose

$$
K=\frac{I Y}{d}
$$

so that $F=1$. This only applies if both switching functions depend on nutrient concentration $C$.

We wish to identify what we mean by starvation conditions, analogously to the criterion (1.2) identified for (1.1). This is less easy, because it depends on the choice of switch functions. If both depend on nutrient concentration $C$ (analogous to autocrine signalling), then $F=1$, but in fact, as we see below, no oscillations appear to occur in this case. 


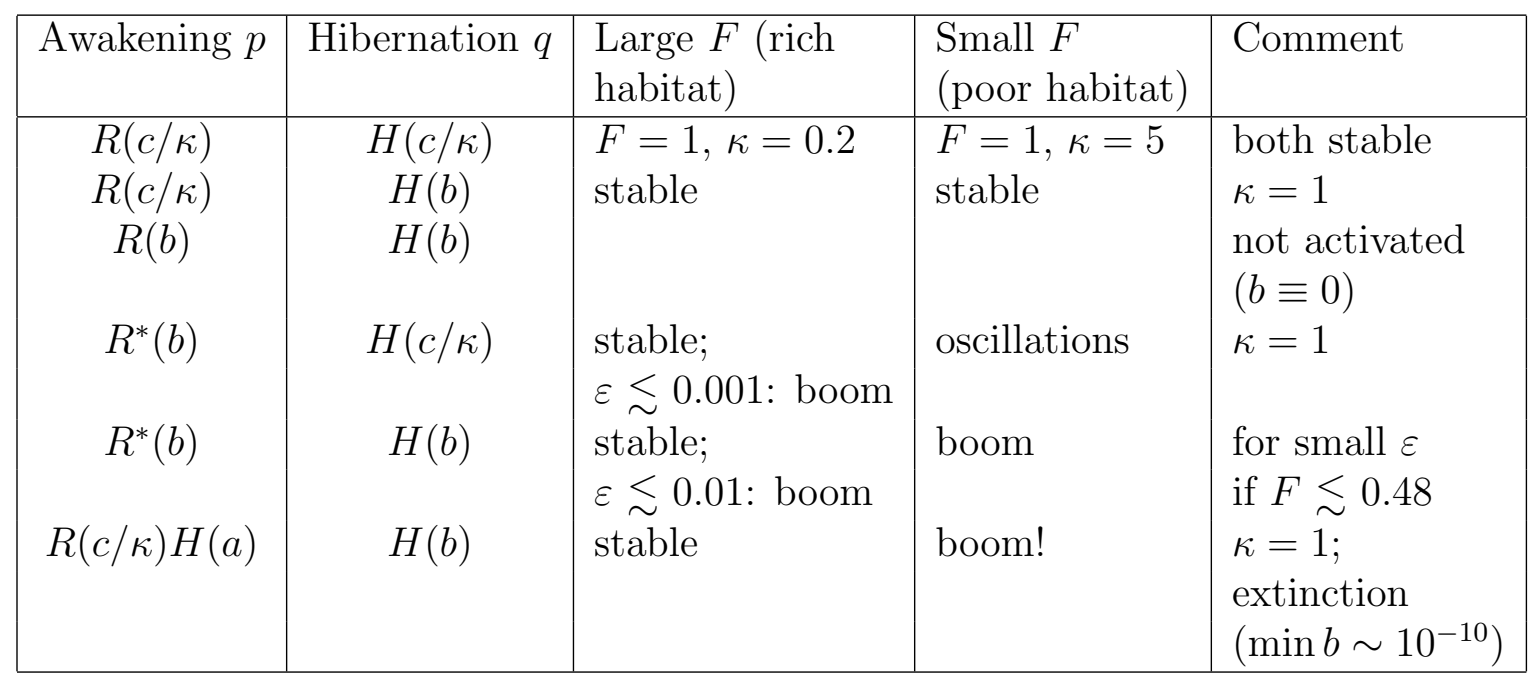

Table 1: Various combinations used in studying the dynamics of (2.3). Unless otherwise stated, we take $F=10$ as the large value and $F=0.5$ as the small value, and $\varepsilon=0.1$ unless stated otherwise. The values of $\mu=\lambda=1$, and the exponents in the Hill functions are $m=n=5$. The value of $\delta=0.1$ in the modified Hill function $R^{*}(x)$. The initial conditions for all runs were $a=c=1, b=0$. The comment "boom" indicates a periodic boom and bust cycle.

Focussing on the case where at least one of the switch functions depends on biomass density $B$, then the scale for $B$ is $K=K_{B}$ and thus

$$
F=\frac{I Y}{d K_{B}}
$$

and we associate starvation conditions with small values of $F$,

$$
I<\frac{d K_{B}}{Y}
$$

At such levels, the steady sustainable microbial population is $b=F$, thus

$$
B=\frac{I Y}{d}
$$

and starvation is associated with such sustainable values of $B$ being less than the value $K_{B}$ where the switching functions become operative.

However, a further complication is that low values of the switching density $K_{B}$ also lead to small values of $\varepsilon$, and these on their own can cause boom and bust cycles, as we will now see.

\section{Boom and bust}

Table 1 shows the various combinations of choices for $p$ and $q$ that we have used. We want $p$ to increase as $b$ or $c$ increase, while $q$ should do the opposite. Our principal 

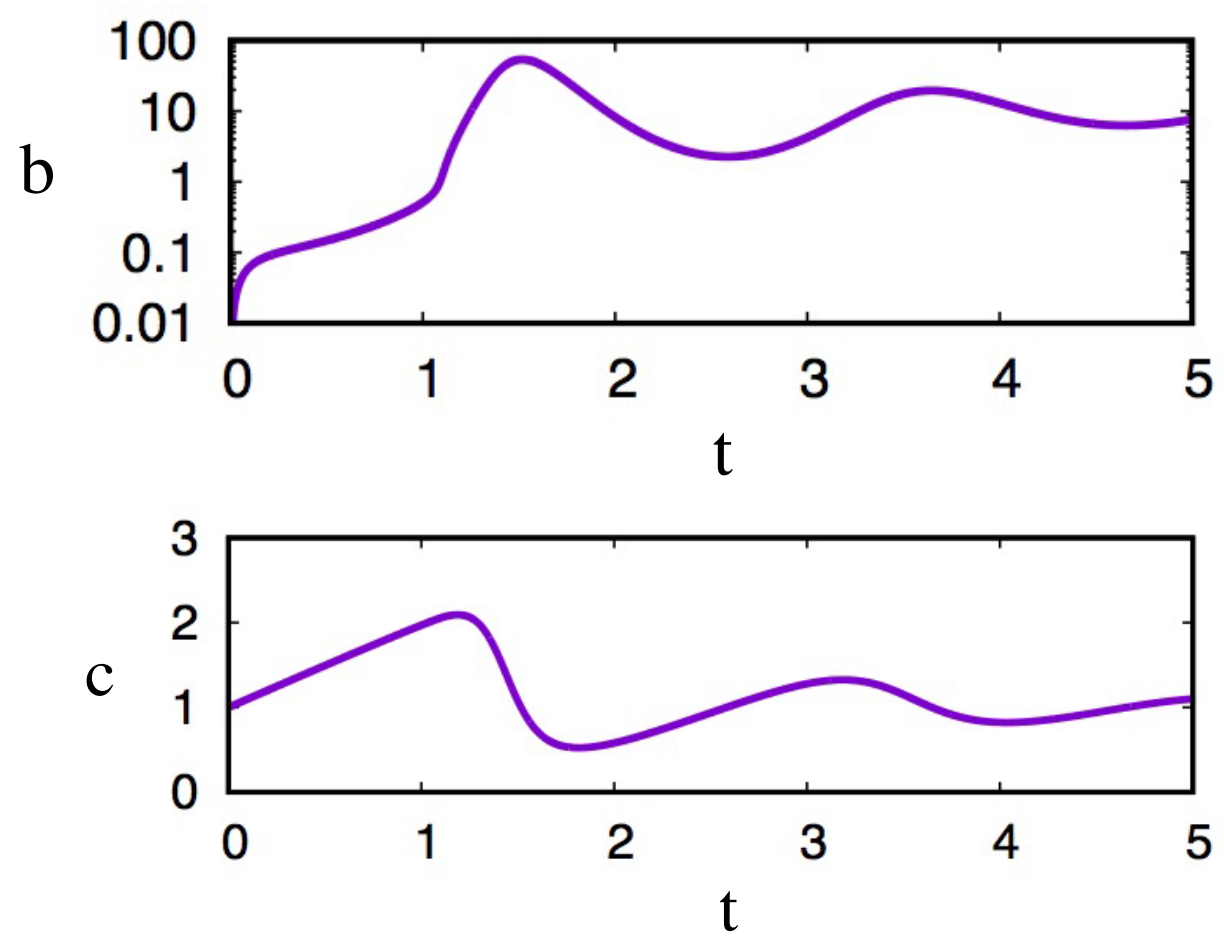

Figure 3: Solution of (2.3) with parameter values $\varepsilon=0.1, \lambda=1, \mu=1, F=10$, with $p=R^{*}(b), \delta=0.1, q=H(b), m=n=5$. The initial conditions are taken to be $a=1, b=0, c=1$ at $t=0$.

choice is whether the microbial activity responds directly to nutrient levels, or whether it is a direct consequence of paracrine signalling from other cells, and thus depends on cell density. The most interesting choice is $p=R^{*}(b), q=H(b)$ (although it makes little difference whether the hibernation function $H$ depends on $c$ or $b$ ), and we focus on this case. Interestingly, it is the dependence of the awakening function which is crucial. Note it is essential to use $R^{*}(b)$ in this case, since otherwise the initial state $b=0$ can not be activated.

In conditions of rich supply, $F \gg 1$, the steady state is usually stable. We then expect $b \sim F \gg 1$, since in the steady state $c=1$. With the switch functions depending on $b, p \approx 1, q \approx 0$, the hibernation switch is inactive, and the model reduces back to the Omta model (1.1). The steady state $c=1, b=F$ is stable, although sufficiently large perturbations cause the approach to the steady state to be relaxational, which is due to the small value of $\varepsilon$. As pointed out by Fowler (2013), when $p=1$ and $\dot{a}=0$ we can put $b=e^{\theta}$ and then $c=1+\varepsilon \dot{\theta}$, so that approximately $\ddot{\theta}+e^{\theta}-F=-\varepsilon \dot{\theta} e^{\theta}$, which is the equation of a weakly damped oscillator. Figure 3 shows a numerical solution of the model (2.3) which shows this behaviour.

However, if $\varepsilon$ is sufficiently small, the minima of $b$ activate the switches, and cause self-sustained boom-and-bust oscillations. Small values of $\varepsilon$ occur when the population rate processes are rapid compared to the response rate of the environment. 

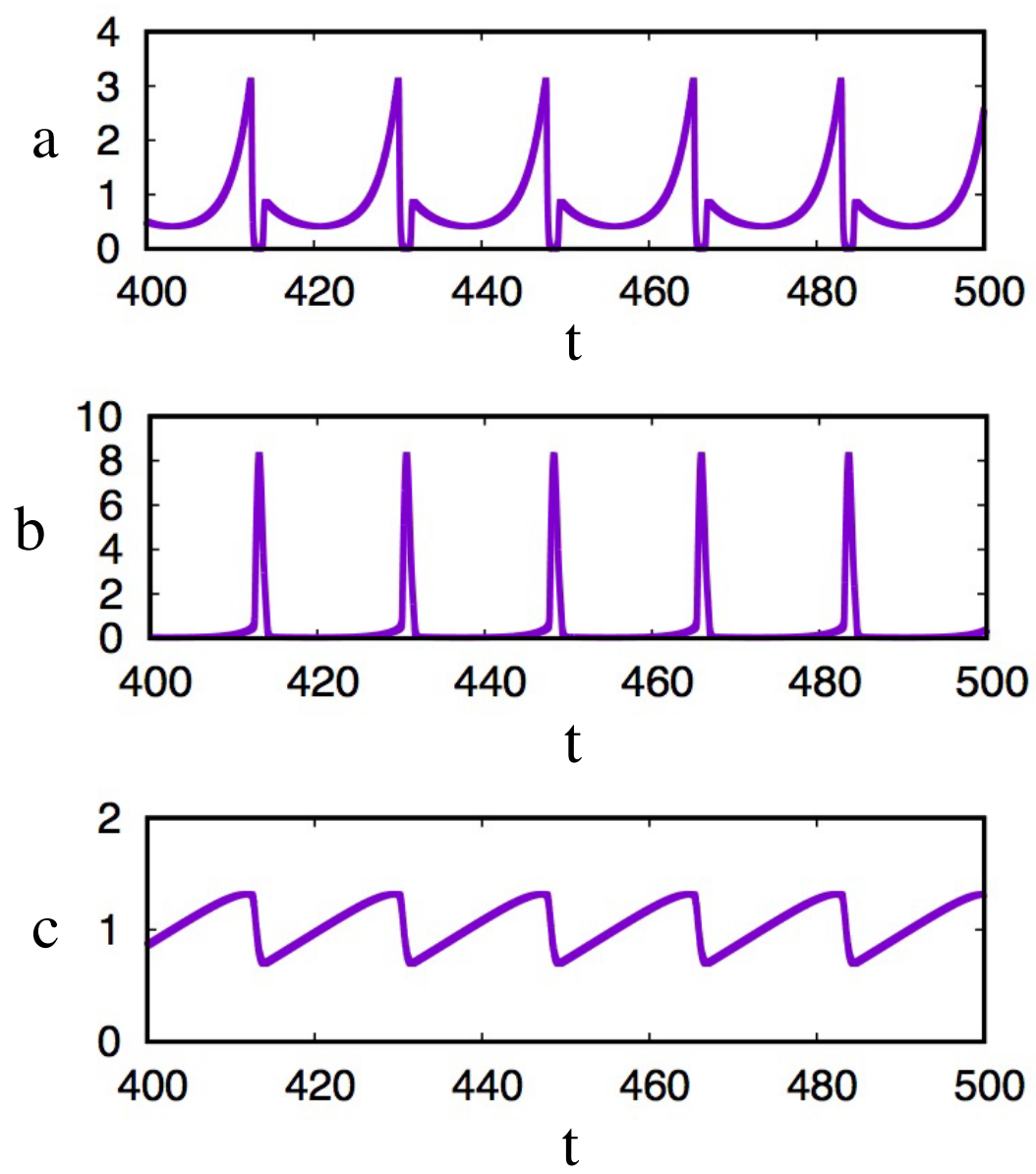

Figure 4: Solution of (2.3). Parameters and initial conditions as for figure 3, except that $F=0.5$.

Relevant values of $\varepsilon$ warrant some discussion. If we focus on the case where one of the switching functions depends on $B$ (which promotes oscillations), then the definition of $\varepsilon$ in $(2.4)$ is

$$
\varepsilon=\sqrt{\frac{k K_{B}}{d}}
$$

where $K_{B}$ is the population level where switching to the dormant state occurs, while $d / k$ is essentially the sustainable population in a well-nourished environment; thus the statement that $\varepsilon \ll 1$ is equivalent to supposing that switching occurs at abnormally low population levels.

If we now reduce $F$ to a starvation level of $O(1)$, such high values of $b$ cannot be maintained. Figure 4 shows a numerical solution for this case. What is interesting about this figure is that the solution settles down to a self-sustained oscillation in which boom-and-bust dynamics occur. However, between the 'blooms', the population becomes largely dormant, but crucially, the microbial population levels do not descend towards extinction. 

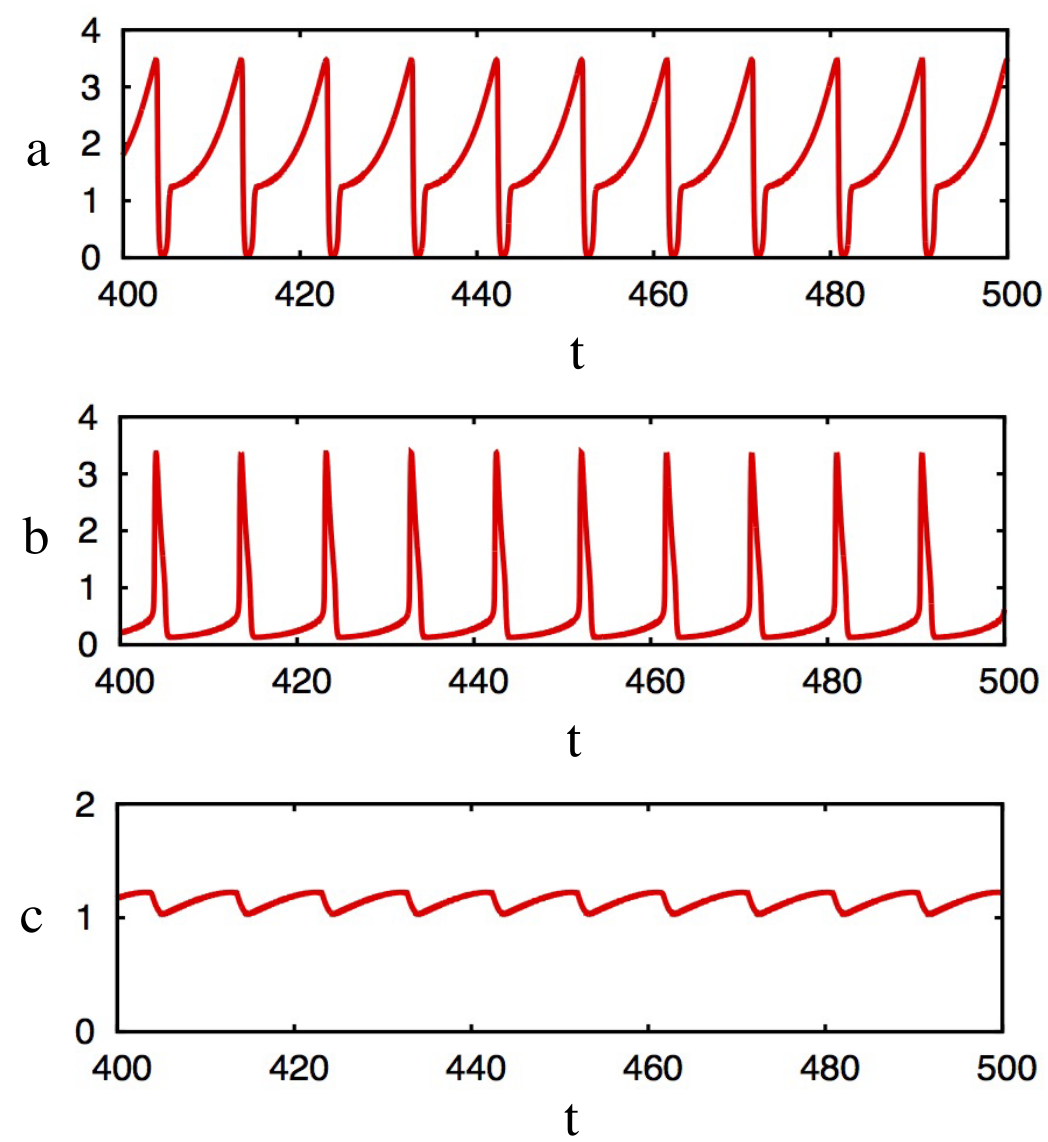

Figure 5: Solution of (2.3). Parameters and initial conditions as for figure 4, except that $\beta=0.1$ (see text for discussion).

Earlier we stated that neglecting the Monod saturation kinetics only had a cosmetic effect; this needs to be quantified. If instead of the linear rate $k B$ in (2.1), we use a Monod term $k B /\left(B+B_{0}\right)$, then the corresponding modification to (2.3) is to replace the term $c$ on the right of the first two equations by $c /(1+\beta b)$, where the new dimensionless parameter is

$$
\beta=\frac{K}{B_{0}} .
$$

For the cases of most interest, $K=K_{B}$ is the population density where switching occurs, and we expect this to be much lower than the saturation value $B_{0}$ where Monod kinetics becomes important. If as a result $\beta \ll 1$, then our earlier neglect is justified. Figure 5 shows the result of a computation similar to figure 4 , but woth $\beta=0.1$; the results are similar. However, if $\beta=1$, the steady state becomes stable.

The self-sustained oscillations suggest that the steady state becomes unstable for $F \lesssim 1$. The steady solution of $(2.3)$ is given by

$$
c=1, \quad b=F, \quad \lambda a=\frac{\mu q b}{p} .
$$


Linearisation about this steady state yields equations with solutions proportional to $e^{\sigma t}$, where $\sigma$ satisfies the cubic

$$
\varepsilon \sigma^{3}+\sigma^{2}\left(M+\lambda p+\varepsilon^{2} F\right)+\varepsilon \sigma F(M+1+\lambda p)+\lambda p F=0,
$$

in which

$$
M=\mu p\left(\frac{q b}{p}\right)^{\prime}
$$

evaluated at the fixed point $b=F$, thus $M=M(F)$. (3.4) has approximate solutions for small $\varepsilon$ of

$$
\sigma \approx-\frac{(M+\lambda p)}{\varepsilon}, \quad \pm\left(-\frac{\lambda p F}{M+\lambda p}\right)^{1 / 2}
$$

and is thus unstable if $M+\lambda p<0$. If $M+\lambda p>0$, the first root is stable and the second pair are, to the next order,

$$
\sigma= \pm i\left(\frac{\lambda p F}{M+\lambda p}\right)^{1 / 2}-\frac{\varepsilon F}{2(M+\lambda p)}\left\{M+1+\lambda p-\frac{\lambda p}{M+\lambda p}\right\}
$$

and thus have positive real part (hence unstable) if

$$
M+(M+\lambda p)^{2}<0 .
$$

Simplifying this, the criterion for instability is that

$$
M(F)<M_{c}(F)=\sqrt{\lambda p+\frac{1}{4}}-\left(\lambda p+\frac{1}{2}\right) .
$$

Although this is an approximate result for $\varepsilon \ll 1$, the exact criterion is quite close: specifically,

$$
M_{c}=\left[\lambda p+\frac{1}{4}\left(1-\varepsilon^{2} F\right)^{2}\right]^{1 / 2}-\left[\lambda p+\frac{1}{2}\left(1+\varepsilon^{2} F\right)\right] .
$$

For the switching functions $p=R^{*}(b)$ and $q=H(b)$, we have $M \approx \frac{\mu}{\delta}$ for $F<1$ and $M \approx 0$ for $F>1$, but $M<0$ when $F \sim 1$. Figure 6 shows the graph of $M-M_{c}$ given by (3.10) as a function of $F$ for the parameter choices in figure 3 . The steady state is oscillatorily unstable for $0.48 \lesssim F \lesssim 1.27$, for the parameter choices in figure 3 , and a bifurcating branch of periodic orbits joins these two critical values. For sufficiently small $\varepsilon$, the upper bifurcating branch is sub-critical, that is to say, boom and bust oscillations are stable together with the steady state, as indicated in table 1 . The lower branch is also sub-critical, although more severe values of $\varepsilon$ appear necessary to obtain this.

\section{Survival in the wild}

We now examine the results of the model in the case where $F=0$. This is of interest, as it may correspond to the most inhospitable environment. According to (3.3), the steady state is then

$$
c=1, \quad b=0, \quad a=0
$$




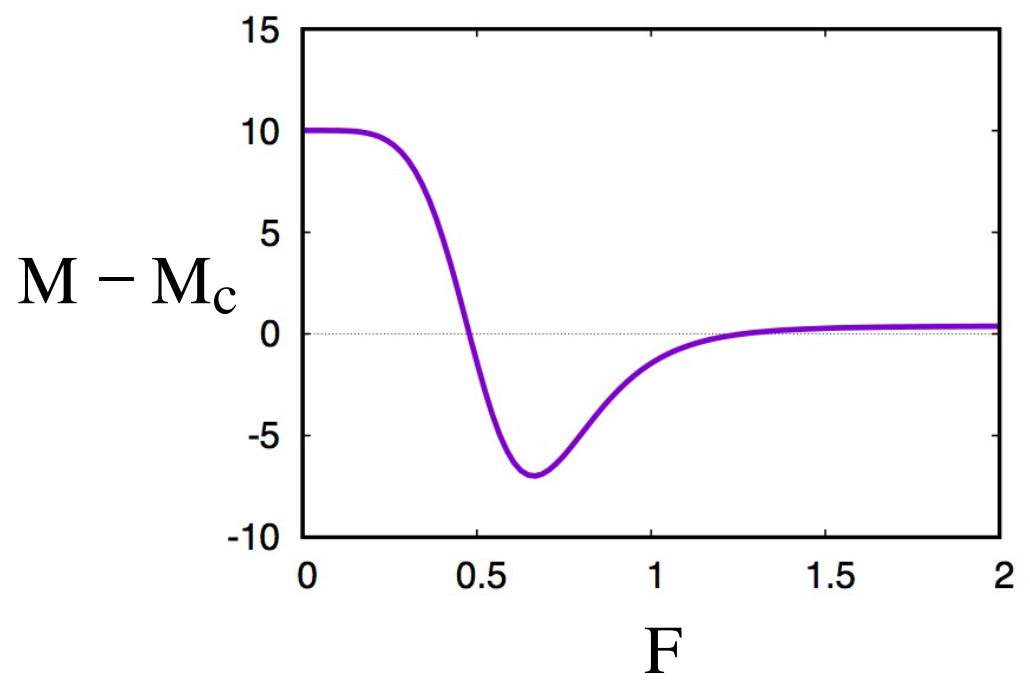

Figure 6: $M(F)-M_{c}(F)$. The steady state is unstable when this is negative, here for $0.48 \lesssim F \lesssim 1.27$, using the parameters in figure 3 .

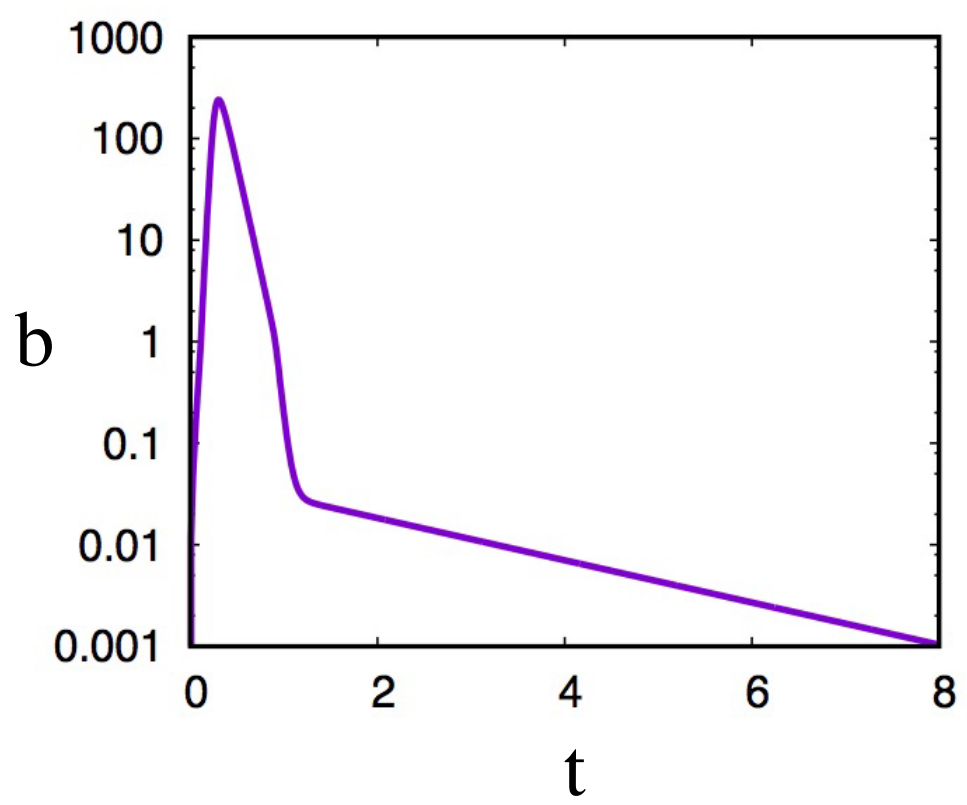

Figure 7: Solution of (2.3) for $b$. Parameters and initial conditions as for figure 3, except that $F=0$ and the initial conditions are $a=1, b=0$ and $c=5$. 
but in fact consultation of (2.3) shows that any value of $c$ will do. With the model (2.3), the extinct state $a=b=0$ is stable. Since $c$ is slowly varying when $\varepsilon \ll 1, a$ and $b$ satisfy the approximate linear equations

$$
\varepsilon\left(\begin{array}{c}
\dot{a} \\
b
\end{array}\right)=\left(\begin{array}{cc}
-\lambda \delta & \mu \\
\lambda \delta & c-1-\mu
\end{array}\right)\left(\begin{array}{l}
a \\
b
\end{array}\right),
$$

and the trace and determinant of the matrix are

$$
T=-\lambda \delta+c-1-\mu, \quad D=-\lambda \delta(c-1) ;
$$

thus while $c>1$ the microbial populations will grow (explosively), but eventually $c<1$, and they decay to zero. This is shown in figure 7 , for example.

Thus our model fails to allow maintenance of a dormant population over long time scales in the absence of a nutrient source. In consideration of this, we return to our original model (2.1), as encapsulated in figure 2. In that figure, an individual bacterium is confronted at low nutrient levels with a choice: to die or to become dormant. However, we assumed that the death rate remained constant. One can argue that this is invalid because a bacterium must do one or the other, so that the death rate should be modulated by a factor $1-q$ (and also $d=r$ so that $\mu=1$ ); in this case the scaled equations (2.3) become

$$
\begin{aligned}
\varepsilon \dot{b} & =(c-1+q) b-\mu q b+\lambda p a \\
\dot{c} & =\varepsilon(F-c b) \\
\varepsilon \dot{a} & =\mu q b-\lambda p a .
\end{aligned}
$$

When $F=0$ and $a$ and $b \rightarrow 0$, we now have $q \approx 1$, and the trace and determinant of the matrix in (4.2) are now

$$
T=-\lambda \delta+c-\mu, \quad D=-\lambda \delta c
$$

so long as $c>0$, the extinct state is locally a saddle. Of course, $c \rightarrow 0$ eventually, and then $T<0$ and $D \rightarrow 0$; this suggests a degenerate extinct state, in which decay (if it occurs) may be algebraic. This is borne out in figure 8 , which shows the result of solving (4.4) with $F=0$. It can be seen that active microbes $b$ (and also the dormant microbes) decay extremely slowly.

Since $\varepsilon \ll 1$, there is evidently a rapid transient of $O(\varepsilon)$ after which

$$
a \approx \frac{\mu q b}{\lambda p}
$$

and in fact this quasi-equilibrium is then maintained indefinitely. (This is consistent with the asymptotic relation $a=10 b$ found numerically for $t>100$, since when $b \ll 1$, $q \approx 1$ and $p \approx \delta=0.1$.)

On a time scale $t \gg 1 / \varepsilon, c \rightarrow 0$, after which (4.4) takes the form

$$
\varepsilon(\dot{a}+\dot{b})=(-1+q) b \approx b^{n+1},
$$




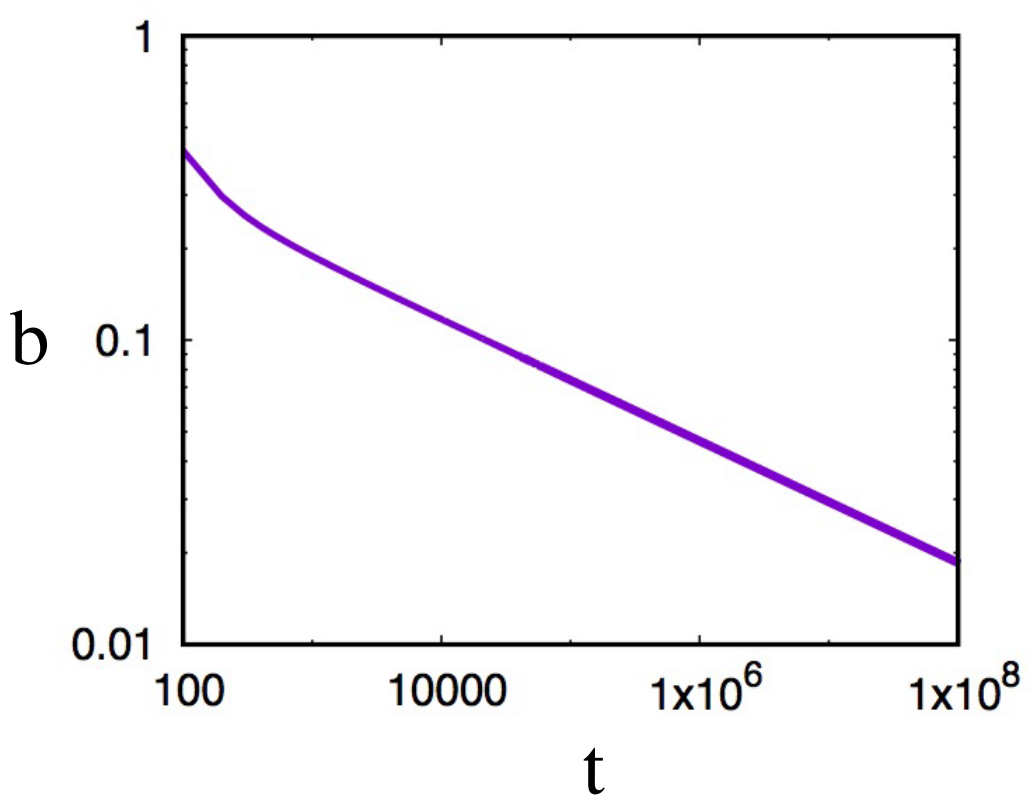

Figure 8: Solution of (4.4) with $F=0$, parameters as in figure 3, and with initial conditions $a=1, b=0.1 c=1$. $c$ reaches zero by about $t=800$, and the algebraic decay of $b$ is closely approximated by $b=0.75 t^{-0.2}$ (and $a=10 b$ ).

since we may take $q \approx 1-b^{n}$ for small $b$. Substituting for $a$ from the quasi-equilibrium (4.6), using also $p \approx \delta$, we obtain

$$
\varepsilon\left(1+\frac{\mu}{\lambda \delta}\right) \dot{b}=-b^{n+1},
$$

with solution

$$
b=\frac{C}{(A+t)^{1 / n}}, \quad C=\left[\frac{\varepsilon}{n}\left(1+\frac{\mu}{\lambda \delta}\right)\right]^{1 / n} ;
$$

for the parameters used in figure 8 , we have $C \approx 0.74$, consistent with the numerical result.

This explains the extremely slow decay of $b$, at least for large $n$, corresponding to a sharp switch. But even for smaller $n$, this leads to extreme longevity. For example, as mentioned in the introduction, we might take a reduction by a factor of $10^{6}$ as representing an extremely hostile environment. Even if $n$ is as low as 2, it takes a dimensionless time of $\sim 10^{12}$ to reach such values. If the cellular time scale is 20 minutes or $10^{3} \mathrm{~s}$, this corresponds to a dimensional time of $10^{15} \mathrm{~s}=30 \mathrm{My}$; a geological time scale. While the devil is in the detail, this shows that the model has the capability of explaining extremely long-lived bacteria in inhospitable conditions.

\section{Discussion}

A variety of mathematical models of dormancy have been proposed in which the switching between active and dormant states is either random or responsive (depen- 
dent on nutrient levels or other indicators of environmental stress). The involvement of paracrine signalling such as quorum sensing has received little attention in the modelling literature. The diversity of dormant states in the microbial world (reviewed by Lennon and Jones (2011)) includes diverse mechanisms of entry to and exit from dormancy. Epstein (2009) argued for the exit from dormancy being dependent on 'scout' cells reactivating randomly to sense the prevailing conditions and then either dying (in adverse conditions) or generating a quorum sensing signal to reactivate other dormant cells. Dworking and Shah (2010) suggested cell growth products such as muropeptides promote reactivation of dormant cells, while Sturm and Dworkin (2015) found phenotypic diversity of spores can provide a mechanism for environment-dependent population regrowth without individuals needing to sense environmental conditions. Meanwhile, there is evidence that quorum sensing may not be involved in the reactivation of antimicrobial-resistant persister cell populations in some experimental systems (Lewis 2007).

While there has been much recent interest in the processes by which bacteria become dormant, and are subsequently revived, our motivation for this study has been to resolve the paradox commonly found in population models, wherein oscillations may occur with extremely low minima, corresponding in practice to extinction. The existence of a dormant phase allows the possibility of such oscillations occurring, without incurring the tiny minima found by Fowler (2014), for example. In this sense, a dormant reservoir of long-lived bacteria resolves the paradox of extinction.

It also provides a vehicle for the understanding of other such situations where oscillatory outbreaks occur. We mentioned two such repetitive outbreaks in the introduction, and our results potentially throw light on these. Plankton blooms are obvious examples of such outbreaks, and are fairly easily interpretable in terms of our theory, the more so because our model actually predicts boom-and-bust oscillations under starvation conditions.

The existence of resting phase reservoirs also provides a potential resolution of the issues concerning extinction of the rabies virus, and indeed generally in terms of epidemics. The issue of extinction in rabies is discussed by Fowler (2000), who suggested a possible resolution in terms of incubation time distributions with long tails, but the notion that the virus persists elsewhere in the environment allows a simpler explanation for repeated outbreaks.

Of particular interest in the model is that in starvation conditions of extreme inhospitality, bacteria can survive for an enormously long time. Inspection of the model shows that this depends on the detail of the assumed probability $q$ of hibernation, and particularly because $1-q$ is very small when $B<K_{B}$. In practical terms, this means that the probability of hibernation becomes very high when the population size decreases below that which triggers the switching to the dormant state.

However, an issue with the model concerns the use of resources. Even when dormant, cells consume resources at a slow rate; worse, if we interpret the equilibrium in (4.6) somewhat analogously to adsorption/desorption kinetics, we would imagine cells continuously cycling between active and dormant states, a process that would require resources. Whether this is the right interpretation of the quasi-equilibrium state in (4.6) is a matter for further perusal; at the least, the issue of resource suggests 
that our discussion of longevity is perhaps only preliminary: but at least it serves to show that a descriptive model is able to produce such longevity as a natural consequence of its form.

\section{Conclusions}

In seeking to resolve the issue of unrealistically low population levels in models of oscillatory microbial or other population growth, we have introduced a model which includes description of a dormant phase. In our model, switching from active to dormant phase and back is associated with a probabilistic switch based either on nutrient or viable microbial density. Particularly, when the re-activation of dormant cells is controlled by microbial density, the model is also capable of explaining the occurrence of self-sustained oscillations of boom-and-bust character similar to plankton blooms, under conditions of low nutrient supply (starvation conditions), with increasingly long intervals between the outbreaks as nutrient is depleted.

Finally, we show that in extremely hostile environments, bacteria can be extremely long-lived if not actually immortal.

\section{Acknowledgements}

This publication has emanated from research conducted with the financial support of Science Foundation Ireland under Grant Number: SFI/09/IN.1/I2645 and SFI/13/IA/1923.

A. C.F. acknowledges the support of the Mathematics Applications Consortium for Science and Industry (www.macsi.ul.ie) funded by the Science Foundation Ireland mathematics grant 12/1A/1683.

\section{References}

Ayati, B.P. 2012 Microbial dormancy in batch cultures as a function of substratedependent mortality. J. Theor. Biol. 293, 34-40.

Ayati, B. P. and I. Klapper 2012 Models of microbial dormancy in biofilms and planktonic cultures. Comm. Math. Sci. 10 (2), 493-511.

Bär, M., J. von Hardenberg, E. Meron and A. Provenzale 2002 Modelling the survival of bacteria in drylands: the advantage of being dormant. Proc. R. Soc. Lond. B269, 937-942.

Campillo, F. and C. Lobry 2012 Effect of population size in a predator-prey model. Ecol. Modell. 246, 1-10.

Cruickshank, I., W.S. C. Gurney and A. R. Veitch 1999 The characteristics of epidemics and invasions with thresholds. Theor. Pop. Biol. 56, 279-292.

Dibrov, B.F., M. A. Livshits and M. V. Volkenstein 1977a Mathematical model of immune processes. J. Theor. Biol. 65, 609-631. 
Dibrov, B.F., M. A. Livshits and M. V. Volkenstein 1977b Mathematical model of immune processes II, kinetic features of antigen-antibody interrelations. J. Theor. Biol. 69, 23-39.

Dworkin, J. and I. M. Shah 2010 Exit from dormancy in microbial organisms. Nature Revs. Microbiol. 8, 890-896.

Epstein, S. S. 2009 Microbial awakenings. Nature 457, 1,083.

Fowler, A. C. 1981 Approximate solution of a model of biological immune responses incorporating delay. J. Math. Biol. 13, 23-45.

Fowler, A. C. 1982 An asymptotic analysis of the logistic delay equation when the delay is large. IMA J. Appl. Math. 28, 41-49.

Fowler, A.C. 2000 The effect of incubation time distribution on the extinction characteristics of a rabies epizootic. Bull. Math. Biol. 62, 633-660.

Fowler, A.C. and M. C. Mackey 2002 Relaxation oscillations in a class of delaydifferential equations. SIAM J. Appl. Math. 63 (1), 299-323.

Fowler, A. C. 2013 Note on a paper by Omta et al. on sawtooth oscillations. SeMA J. 62, 1-13.

Fowler, A. C. 2014 Starvation kinetics of oscillating microbial populations. Math. Proc. R. Ir. Acad. 114 (2), 173-189.

Fowler, A. C., H. F. Winstanley, M. J. McGuinness and L. B. Cribbin 2014 Oscillations in soil bacterial redox reactions. J. Theor. Biol. 342, 33-38.

Goldbeter, A. 1996 Biochemical oscillations and cellular rhythms. C. U.P., Cambridge.

Hadeler, K. P. 2013 Quiescence, excitability, and heterogeneity in ecological models. J. Math. Biol. 66, 649-684.

Harms, A., E. Maisonneuve and K. Gerdes 2016 Mechanisms of bacterial persistence during stress and antibiotic exposure. Science, 354 (6318), aaf4268.

Haurie, C., M. C. Mackey and D. C. Dale 1998 Cyclical neutropenia and other periodic hematological diseases: a review of mechanisms and mathematical models. Blood 92, 2629-2640.

He, Y., J. Yang, Q. Zhuang, J. W. Harden, A. D. McGuire, Y. Liu, G. Wang and L. Gu 2015 Incorporating microbial dormancy dynamics into soil decomposition models to improve quantification of soil carbon dynamics of northern temperate forests. J. Geophys. Res: Biogeosciences 120 (12), 2596-2611.

Hoehler, T. M. and B. B. Jørgensen 2013 Microbial life under extreme energy limitation. Nature Rev. Microbiol. 11 (2), 83-94. 
Huppert, A., B. Blasius, R. Olinky and L. Stone 2005 A model for seasonal phytoplankton blooms. J. Theor. Biol. 236 (3), 276-290.

Joergensen, R. G. and F. Wichern 2018 Alive and kicking: Why dormant soil microorganisms matter. Soil Biology and Biochemistry 116, 419-430.

Jones, S.E. and J. T. Lennon 2010 Dormancy contributes to the maintenance of microbial diversity. Proc. Nat. Acad. Sci. 107, 5,881-5,886.

Kaprelyants, A. S., J. C. Gottschal and D. B. Kell 1993 Dormancy in non-sporulating bacteria. FEMS Microbiol. Revs. 104, 271-286.

Kirchman, D. L. 2012 Processes in microbial ecology. O. U. P., Oxford.

Lennon, J. T. and S. E. Jones 2011 Microbial seed banks: the ecological and evolutionary implications of dormancy. Nature Rev. Microbiol. 9, 119-130.

Lipworth, S., R. J. H. Hammond, V. O. Baron, Y. Hu, A. Coates and S. H. Gillespie 2016 Defining dormancy in mycobacterial disease. Tuberculosis 99, 131-142.

Locey, K. J., M. C. Fisk and J. T. Lennon 2017 Microscale insight into microbial seed banks. Frontiers in Microbiology 7, 2040.

Mackey, M.C. 1978 A unified hypothesis for the origin of aplastic anaemia and periodic haematopoesis. Blood 51, 941-956.

Mahadevan, A., E. D'Asaro, M.-J. Perry and C. Lee 2012 Eddy-driven stratification initiates North Atlantic Spring phytoplankton blooms. Science 337 (6090), $54-58$.

Malik, T. and H. Smith 2006 A resource-based model of microbial quiescence. J. Math. Biol. 53, 231-252.

Malik, T. and H. Smith 2008 Does dormancy increase fitness of bacterial populations in time-varying environments? Bull. Math. Biol. 70, 1,140-1,162.

Mollison, D. 1991 Dependence of epidemic and population velocities on basic parameters. Math. Biosci. 107, 255-287.

Murray, J.D., E. A. Stanley and D. L. Brown 1986 On the spatial spread of rabies among foxes. Proc. R. Soc. Lond. B229, 111-150.

Omta, A. W., G. A. K. van Voorn, R. E. M. Rickaby and M. J. Follows 2013 On the potential role of marine calcifiers in glacial-interglacial dynamics. Global Biogeochem. Cycles 27, 692-704.

Renshaw, E. 2005 Epidemic models, spatial. In: Encyclopedia of biostatistics, 2nd ed., eds. P. Armitage and T. Colton, vol. 3, pp. 1,693-1,699. John Wiley, Chichester. 
Rittershaus, E. S. C. and S.-H. Baek and C. M. Sassetti 2013 The normalcy of dormancy: common themes in microbial quiescence. Cell Host \& Microbe 13 (6), 643-651.

Roszak, D. B. and R. R. Colwell 1987 Survival strategies of bacteria in the natural environment. Microbiol. Revs. 51, 365-379.

Sanchez-Vila, X., S. Rubol, A. Carles-Brangari and D. Fernàndez-Garcia 2013 An analytical solution to study substrate-microbial dynamics in soils. Adv. Water Resour. 54, 181-190.

Shoemaker, W. R. and J. T. Lennon 2018 Evolution with a seed bank: the population genetic consequences of microbial dormancy. Evolutionary Applications 11, 6075 .

Stevenson, L. H. 1978 A case for bacterial dormancy in aquatic systems. Microb. Ecol. 4, 127-133.

Stolpovsky, K., I. Fetzer, P. van Cappellen and M. Thullner 2016 Influence of dormancy on microbial competition under intermittent substrate supply: insights from model simulations. FEMS Microbiol. Ecol. 92 (6), fiw071.

Stolpolsky, K., P. Martinez-Lavanchy, H. J. Heipieper, P. van Cappellen and M. Thullner 2011 Incorporating dormancy in dynamic microbial community models. Ecol. Modell. 222, 3,092-3,102.

Sturm, A. and J. Dworkin 2015 Phenotypic diversity as a mechanism to exit cellular dormancy. Current Biology, 25 (17), 2272-2277.

Wang, G., M. A. Mayes, L. Gu and C. W. Schadt 2014 Representation of dormant and active microbial dynamics for ecosystem modeling. PloS one 9 (2), e89252.

Wang, J.D. and P.A. Levin 2009 Metabolism, cell growth and the bacterial cell cycle. Nature Revs. Microbiol. 7 (11), 822-827. 